\title{
Effect of Sodium Fluorescein and Plating Medium on Recovery of Irradiated Escherichia coli and Serratia marcescens from Aerosols
}

\author{
EMERSON L. DORSEY, RICHARD F. BERENDT, AND EVERETT L. NEFF, JR. \\ Aerobiology and Evaluation Laboratories, Fort Detrick, Frederick, Maryland 21701
}

Received for publication 23 July 1970

\begin{abstract}
Irradiation of aerosols of either Escherichia coli or Serratia marcescens with simulated solar (xenon) radiation caused a significant decrease in viability. When sodium fluorescein was employed to determine the physical loss of organisms from the aerosol, an additional adverse effect upon survival was noted. The decay curves indicated that at least two mechanisms of inactivation were operative, one due to aerosolization, the other to irradiation. After collection from aerosols, both species of microorganisms grew better on blood agar base than on Casitone agar, but this finding did not appear to be related to the effect of irradiation.
\end{abstract}

When microorganisms are disseminated into aerosol chambers and samples are collected at periodic intervals thereafter, the viable concentration of cells decreases (total decay). Total decay is the sum of two components: biological decay, which is a decrease in number due to death or inactivation of cells, and physical decay, a decrease in number due to impingement upon surfaces and to settling of aerosol particles containing organisms. To study the biological reactions that occur in aerosolized microorganisms, it is necessary to determine what fraction of the total decrease in organism numbers is due to physical decay. The difference between these values then represents biological decay. To measure physical decay, tracers such as radioactive cells $(4)$ or fluorescent dyes $(7,9)$ are commonly employed. Sodium fluorescein has been used in this laboratory as a physical tracer for a number of years.

Recently, a laboratory was constructed to study the effect of simulated or natural solar radiation upon airborne microorganisms. In keeping with the necessity of distinguishing between the biological effects of radiation and physical loss, we have studied the possible toxicity of sodium fluorescein as a physical tracer for airborne Serratia marcescens and Escherichia coli in an irradiated environment. A recent review by Foote (2) has emphasized the necessity for this kind of study because fluorescent compounds may become toxic for biological systems in such an environment.

In addition, preliminary investigation indicated that a greater number of irradiated $S$. marcescens recovered from aerosols multiplied on blood agar base than on Casitone agar. Consequently, the scope of the investigation was enlarged to include study of the effect of these two plating media.

\section{MATERIALS AND METHODS}

Aerosol chamber and associated equipment. A heliostat-revolving drum system was made to study irradiated aerosols. The use of the revolving drum for the study of aerosols has been described by Goldberg et al. (3). The essential components of the system are shown in Fig. 1. The aerosol chamber was a revolving dodecagon about $4 \mathrm{ft}$ in diameter and $2 \mathrm{ft}$ long with a volume of 650 liters, enclosed in a safety cabinet system. Each of the 12 surfaces of the periphery consisted of panes of 7910 Vycor glass (Corning Glass Co., Corning, N.Y.) that permitted the transmission of virtually all radiation wavelengths from 300 to $2,500 \mathrm{~nm}$. The drum could be operated at any one of six preset speeds, ranging from 2 to $7 \mathrm{rev} / \mathrm{min}$. Dissemination and sampling of aerosols and measurement of the physical variables of temperature, dew point, and light intensity were carried out through appropriate sampling lines in one of the 14-inch hubs of the drum. Six 4-inch ports were drilled in the other hub plate to permit animal exposure. Temperature was controlled by the temperature of the cabinet in which the drum was situated, and the internal temperature of the aerosol drum was continuously recorded.

Provisions for studying both simulated and natural solar radiation have been made with this system. Simulated solar radiation was provided by a $10-\mathrm{kw}$ xenon lamp (Osram Gesellschaft, Berlin, West Germany) mounted beneath the drum. A Pyrex plate 
mounted directly above the light cut off emissions below $300 \mathrm{~nm}$, the shortest wavelength that has been found at the earth's surface (6). A five-position switch permitted a choice of total light intensities ranging from about 500 to $1,300 \mathrm{mcal} / \mathrm{cm}^{2} / \mathrm{min}$. A representative energy distribution curve for the xenon light is shown in Fig. 2.

Natural sunlight was directed into the drum through several layers of Vycor glass from a fixed, frontsurface mirror mounted on a platform fastened to the roof of the building. The fixed mirror reflected an image directed into it from a 5- by 10 -ft front-surface tracking mirror that was also mounted on the platform. The tracking mirror and the equipment to operate it constituted the heliostat system. The heliostat

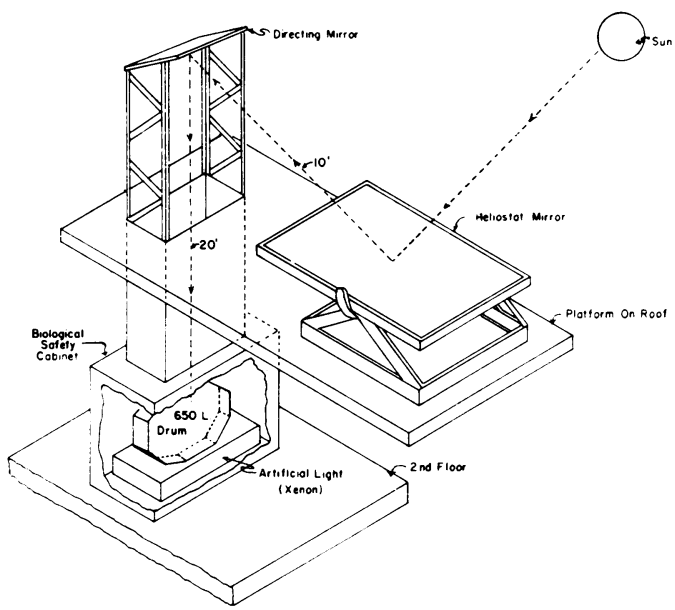

FIG. 1. Solar radiation heliostat and revolving drum system. was constructed so that, after initial adjustment for elevation and azimuth, the mirror would track the sun across the sky. After initial adjustment, the entire apparatus could be controlled from a console located in the drum room. Additional control of light intensity could be achieved by tracking slightly in front of or behind the sun, and both the heliostat and xenon light systems were provided with light attenuation screens of various meshes to achieve greater control of light intensity.

All manipulations associated with dissemination, sampling, and routine assay procedures were carried out within the sealed safety cabinet surrounding the drum.

Light-sensing equipment. Light intensity, in the near-ultraviolet range, was measured with a sensor consisting of an RCA-935 phototube mounted behind a Corning 7-54 filter. This filter had maximum transmittance from 300 to $350 \mathrm{~nm}, 50 \%$ transmittance at $385 \mathrm{~nm}, 10 \%$ at $402 \mathrm{~nm}$, and none at $420 \mathrm{~nm}$. The sensor, connected to a microammeter, was calibrated by the National Bureau of Standards.

Aerosol generation and sampling. Aerosols were generated in the revolving drum with a FK-8 gun (5) and sampled with AGI-20 impingers (5) operating at 6 liters $/ \mathrm{min}$, each containing $20 \mathrm{ml}$ of phosphate buffer $(p H$ 7.2). The relative humidity, after dissemination, was $60 \%$; the temperature was $21.1 \mathrm{C}$.

Culture preparation. Cultures of the Porton strain 162 of $E$. coli were prepared by thawing frozen concentrated pellets prepared by centrifugation of Tryptose broth cultures and resuspending them in gelatinphosphate buffer. The pellets had been stored in a dry-ice box for approximately 4 years. The reconstituted cultures had a concentration of about $10^{10}$ viable cells per $\mathrm{ml}$.

Cultures of the $8 \mathrm{UK}$ strain of $S$. marcescens were grown in Tryptose broth (Difco) at $22 \mathrm{C}$ for 16 to 18

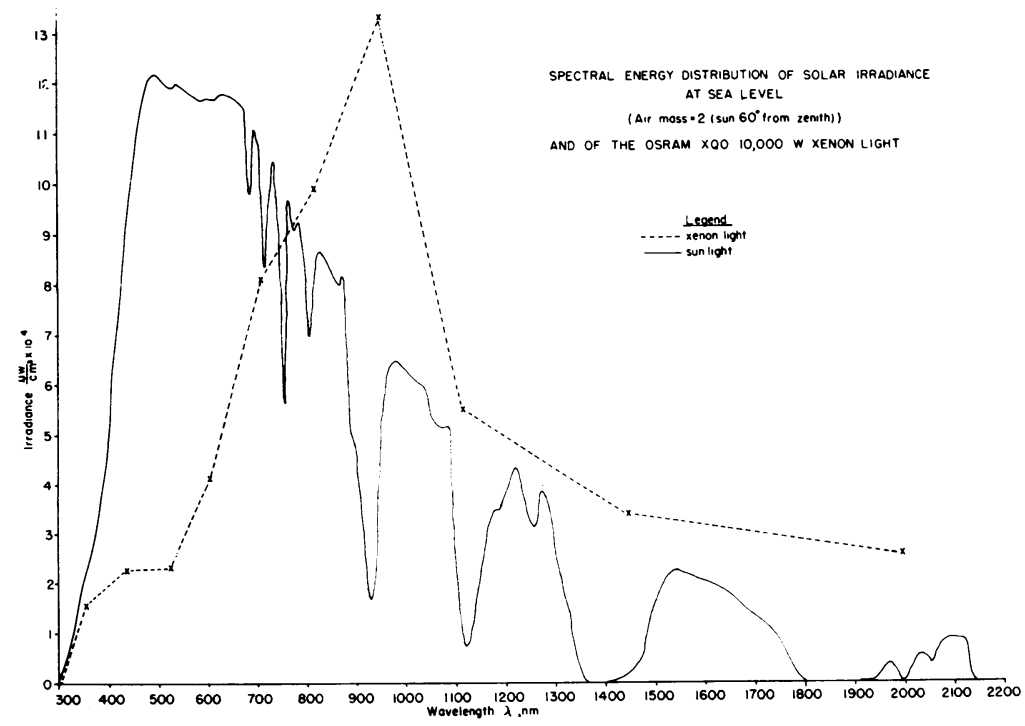

FIG. 2. Representative spectral energy distribution curves for natural and simulated solar radiation. 
TABLE 1. Effect of blood agarbase and Casitone agars, of sodium fluorescein tracer, and of simulated solar radiation on survival of airborne $S$. marcescens

\begin{tabular}{|c|c|c|c|c|c|}
\hline \multirow{3}{*}{$\begin{array}{l}\text { Sampling } \\
\text { time (min) }\end{array}$} & \multirow{3}{*}{$\begin{array}{c}\text { Light } \\
\text { intensity } \\
\text { (mcal/ } \\
\mathrm{cm}^{2} / \mathrm{min} \text { ) }\end{array}$} & \multicolumn{4}{|c|}{ Per cent recovery with indicated medium ${ }^{a}$} \\
\hline & & \multicolumn{2}{|c|}{ Blood agar base } & \multicolumn{2}{|c|}{ Casitone agar } \\
\hline & & Uranine & No uranine & Uranine & No uranine \\
\hline 2 & 0 & $48.9(46)^{b}$ & $53.9(40)$ & $46.4(40)$ & $49.6(37)$ \\
\hline 4 & & $48.8(41)$ & $56.5(39)$ & $45.4(37)$ & $51.9(39)$ \\
\hline 8 & & $44.5(40)$ & $49.2(41)$ & $41.2(37)$ & $44.6(36)$ \\
\hline 16 & & $34.9(45)$ & $41.8(36)$ & $30.5(44)$ & $36.8(37)$ \\
\hline 32 & & $24.9(54)$ & $33.2(35)$ & $22.2(52)$ & $29.6(35)$ \\
\hline 64 & & $14.1(51)$ & $16.9(23)$ & $12.0(50)$ & $14.9(24)$ \\
\hline 2 & 32 & $45.7(27)$ & $41.7(41)$ & $44.4(23)$ & $40.6(36)$ \\
\hline 4 & & $40.8(32)$ & $42.2(29)$ & $38.6(31)$ & $38.3(33)$ \\
\hline 8 & & $34.0(35)$ & $30.9(31)$ & $31.3(40)$ & $26.9(30)$ \\
\hline 16 & & $18.5(30)$ & $21.3(26)$ & $15.5(26)$ & $17.2(18)$ \\
\hline 32 & & $3.6(44)$ & $8.4(35)$ & $3.1(49)$ & $6.9(36)$ \\
\hline 64 & & $0.05(89)$ & $0.4(36)$ & $0.03(116)$ & $0.4(50)$ \\
\hline 2 & 70 & $32.6(35)$ & $49.6(41)$ & $30.5(36)$ & 46.9 (44) \\
\hline 4 & & $27.4(42)$ & $37.5(26)$ & $24.4(47)$ & $34.3(22)$ \\
\hline 8 & & $18.1(48)$ & $25.5(23)$ & $15.4(47)$ & $23.5(20)$ \\
\hline 16 & & $3.6(41)$ & $11.6(35)$ & $2.5(46)$ & $10.0(41)$ \\
\hline 32 & & $0.1 \quad(80)$ & $1.0(44)$ & $0.08 \quad(89)$ & $0.9(45)$ \\
\hline 64 & & $0.00006(160)$ & $0.009(63)$ & $0.00003(175)$ & 0.007 (69) \\
\hline
\end{tabular}

${ }^{a}$ Mean of six runs for each condition.

${ }^{b}$ Figures in parentheses are coefficients of variation rounded off to the nearest whole number.

TABLE 2. Effect of blood agar base and Casitone agars, of sodium fluorescein tracer, and of simulated solar radiation on survival of airborne $E$. coli

\begin{tabular}{|c|c|c|c|c|c|}
\hline \multirow{3}{*}{$\begin{array}{c}\text { Sampling } \\
\text { time (min) }\end{array}$} & \multirow{3}{*}{$\begin{array}{l}\text { Light } \\
\text { intensity } \\
\text { (mcal/ } \\
\mathrm{cm}^{2} / \mathrm{min} \text { ) }\end{array}$} & \multicolumn{4}{|c|}{ Per cent recovery with indicated medium ${ }^{a}$} \\
\hline & & \multicolumn{2}{|c|}{ Blood agar base } & \multicolumn{2}{|c|}{ Casitone agar } \\
\hline & & Uranine & No uranine & Uranine & No uranine \\
\hline 2 & 0 & $25.3(17)^{b}$ & $22.1(14)$ & $22.2(17)$ & $21.6(20)$ \\
\hline 4 & & $18.4(13)$ & $17.1(19)$ & $15.7(13)$ & $14.7(19)$ \\
\hline 8 & & $11.5(22)$ & $12.8(19)$ & $9.5(15)$ & $11.2(23)$ \\
\hline 16 & & $9.0(22)$ & $10.1(13)$ & $7.3(27)$ & $9.2(12)$ \\
\hline 32 & & $5.1(48)$ & $7.7(26)$ & $4.5(51)$ & $6.6(30)$ \\
\hline 64 & & $1.8(43)$ & $4.0(26)$ & $1.5(61)$ & $3.5(23)$ \\
\hline 2 & 32 & $15.4(20)$ & $15.6(17)$ & $12.6(17)$ & $12.9(25)$ \\
\hline 4 & & $9.0(15)$ & $11.4(29)$ & $6.8(17)$ & $8.6(41)$ \\
\hline 8 & & $3.4(37)$ & 7.1 (35) & $2.6(36)$ & $5.4(50)$ \\
\hline 16 & & $0.5(46)$ & $3.2(47)$ & $0.3(59)$ & $2.4(60)$ \\
\hline 32 & & $0.004(60)$ & $0.9(61)$ & $0.001 \quad(86)$ & $0.6(80)$ \\
\hline 64 & & $\mathbf{N R}^{c}$ & 0.04 & NR & $0.02(91)$ \\
\hline 2 & 70 & $8.7(27)$ & $9.9(24)$ & $7.4(21)$ & $7.9(19)$ \\
\hline 4 & & $3.5(48)$ & $6.1(30)$ & $2.7(57)$ & $4.9(30)$ \\
\hline 8 & & $0.3(79)$ & $2.7(40)$ & $0.2(63)$ & 2.1 (47) \\
\hline 16 & & 0.002 (74) & 0.7 (49) & $0.001 \quad(133)$ & $0.5(52)$ \\
\hline 32 & & NR & $0.04(62)$ & NR & $0.03(65)$ \\
\hline 64 & & NR & $0.0001 \quad(171)$ & NR & $0.00007(217)$ \\
\hline
\end{tabular}

${ }^{a}$ Mean of six runs for each condition.

${ }^{b}$ Figures in parentheses are coefficients of variation rounded off to the nearest whole number.

${ }^{c} \mathrm{NR}=$ no recovery. 
hr. Viable concentration was about $10^{10}$ per $\mathrm{ml}$. This organism was disseminated from the growth medium. A 1-ml amount of culture was disseminated for each experiment.

Experimental design. A four-factor factorial experiment (with split plot characteristics) was used for the study of each organism. Randomization involved three levels of simulated solar radiation intensity $\left(0,32\right.$, and $\left.70 \mathrm{mcal} / \mathrm{cm}^{2} / \mathrm{min}\right)$, two concentrations of uranine ( 0 and $1.0 \mathrm{mg} / \mathrm{ml}$ of culture) and two agar media [Casitone and blood agar base (Difco)]. The blood agar base contained added glucose $(0.1 \%)$ and sodium sulfite $(0.004 \%)$. Cycloheximide $(0.01 \%)$ was added to each medium to inhibit growth of molds.

Aerosols were sampled for $1 \mathrm{~min}$ at 2, 4, 8, 16, 32, and $64 \mathrm{~min}$ after dissemination. Each experiment was replicated six times. Each sample was plated upon both media after appropriate dilution and the various light-tracer combinations were determined from separate aerosols, so it was expected that the analysis would be more sensitive to medium differences than to radiation, tracer effects, or both.

\section{RESULTS}

The mean per cent recovery values and their respective coefficients of variation are given in Tables 1 and 2 for $S$. marcescens and $E$. coli, respectively. The comparison of the effects of the test variables on the recoveries was based upon formal tests of significance. The recoveries of both species were significantly higher on blood agar base than upon Casitone agar $(P \leq 0.01)$ for both irradiated and nonirradiated aerosolized organisms. No difference in concentration attributable to plating medium was seen with nonaerosolized cells.

The deleterious effects of irradiation were also highly significant $(P \leq 0.01)$ for all conditions of test except for the $S$. marcescens at $2 \mathrm{~min}$ after dissemination. There was also a significant difference between the two intensities of irradiation for most conditions. Sodium fluorescein tracer was found to affect recoveries of both species significantly, most strongly at the highest light intensity $\left(70 \mathrm{mcal} / \mathrm{cm}^{2} / \mathrm{min}\right)$. Also, the effect of the dye was more marked at 16, 32, and $64 \mathrm{~min}$ than at 2,4 , and $8 \mathrm{~min}$. The statistical analysis was complicated by either very low or complete absence of recovery of cells suspended in sodium fluorescein 64 min after dissemination. Even in the cases in which cells were recovered, the variation at this time was very high, and the analysis was correspondingly less sensitive.

Another difference that was noted was the effect of light on the shape of the decay curve. A plot of the log per cent recovery against time for $S$. marcescens is shown in Fig. 3 and for $E$. coli in Fig. 4. Aerosols of non-irradiated organisms generally show a decreasing decay rate as time progresses (upward concavity), but irradi-

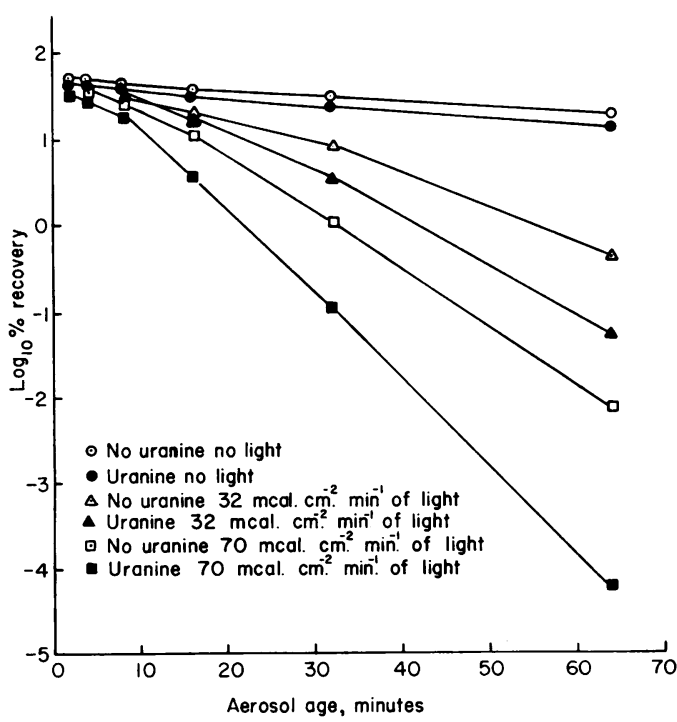

FIG. 3. Effect of tracer and irradiation on the recovery of S. marcescens from aerosols (assayed on blood agar base).

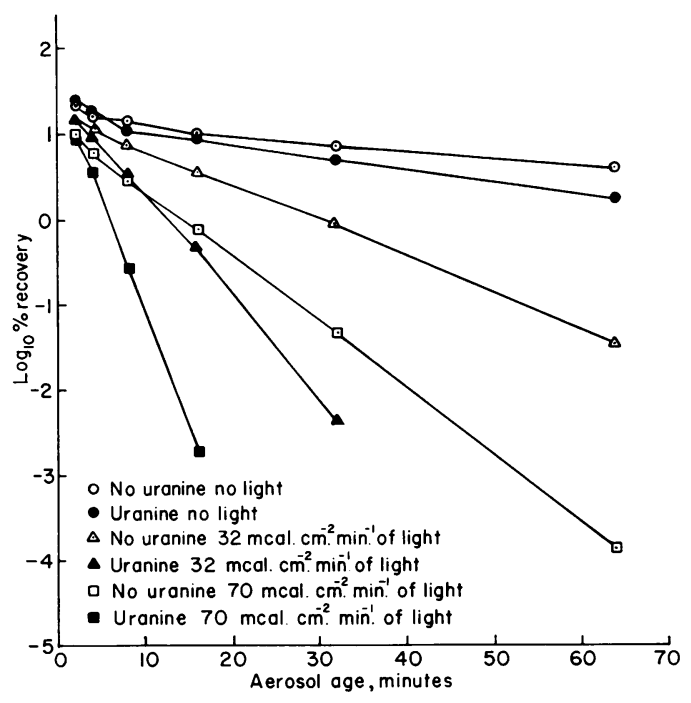

FIG. 4. Effect of tracer and irradiation on the recovery of E. coli from aerosols (assayed on blood agar base).

ated airborne cells seem to be inactivated at an increasing rate with time (downward concavity). This phenomenon was noted with both microorganisms.

\section{DISCUSSION}

The major objective of this study was to determine the adequacy of sodium fluorescein as a 
physical tracer for aerosols of irradiated $S$. marcescens and $E$. coli. The data presented conclusively demonstrate that it is not suitable for this purpose.

The adverse effect of fluorescein dye is important for both practical and theoretical reasons. Practically, it will be necessary to develop a physical tracing technique that does not require the use of a photosensitive compound, a problem that is presently under study. Theoretically, the phenomenon of photosensitivity is of great importance. Since microorganisms are killed by sunlight, it is obvious that certain compounds or systems within them absorb radiation. In the case of ultraviolet irradiation, nucleic acids seem to be the compounds that absorb the radiation and thymine polymers are the toxic materials produced (1). The compounds that absorb longer wavelengths have not been studied as intensely, however, and will be investigated. It is also important to study the menstruum in which microorganisms are suspended whether artificially disseminated as in aerosol experimentation or naturally, as in sneezing or coughing. Sunlight may react with components of the suspending material to produce effects that may not occur under other conditions. A great many biologically active compounds have been shown to be photosensitive (2). In some cases, the result may not be as obvious as cell death; mutation may be induced or changes in infectivity may take place.

The findings demonstrate that the decay of aerosolized irradiated organisms results from factors supplementary to those causing inactivation of nonirradiated cells. The difference in mechanism is best illustrated by comparison of the decay patterns of irradiated and nonirradiated cells. The downward concavity of the recovery curves of irradiated organisms suggests that a minimum cumulative amount (threshold) of radiation must be absorbed before cells react to irradiation. The threshold seems to be achieved more rapidly in $E$. coli than in $S$. marcescens. The pattern of decay of irradiated cells has been discussed by Webb (8), who also stresses the effect of relative humidity, a variable not investigated in this laboratory to date.

The rapid decay of irradiated airborne organisms suggests a need for new mathematical approaches and for careful preliminary study in order that samples may be obtained as long as possible after dissemination. The commonly used simple exponential model, $\mathrm{C}_{\mathrm{t}}=\mathrm{C}_{0} e^{\mathrm{tk}}$, is not always adequate for description of decay curves that may be sigmoidal.

Simulated-solar radiation was used in this study. A problem, presently under investigation, is a comparison of the effect of solar radiation with that of the xenon light (simulated-solar radiation) under various environmental conditions. Xenon has intensity peaks in the longer wavelengths ( 800 to $1,000 \mathrm{~nm}$ ) that are not found in natural light, but careful evaluation of the action spectra of the two kinds of radiation will be necessary to determine whether these peaks have significant antimicrobial activity.

The recovery of both organisms was significantly higher on blood agar base than upon Casitone agar. The enhancement by blood agar seems to be more closely related to aerosol stress than to radiation since the increase in recovery was the same for the dark control as for the irradiated aerosols, and no effect of plating medium was found with nonaerosolized organisms. Continued investigation of the effect of plating medium may provide information relating to the mechanism of aerosol inactivation, but, in this case at least, it would not explain the action of irradiation.

\section{ACKNOWLEDGMENTS}

The authors express their gratitude to N. R. Bohidar, C-E-I-R, Inc., for statistical design, analyses, and interpretation of the data and to Leonard Wilhelm, Charles Veirtz, Henry Wallace, Pearl Ambush, Elinor Brown, Catherine Mogg, and Ethel Dolan for technical assistance.

\section{LITERATURE GITED}

1. Beukers, R., and W. Berends. 1960. Isolation and identification of the irradiation product of thymine. Biochim. Biophys. Acta $41: 550-551$.

2. Foote, C. S. 1968. Mechanism of photosensitized oxidation. Science 162:963-970.

3. Goldberg, L. J., H. M. S. Watkins, E. E. Boerke, and M. A. Chatigny. 1958. The use of a rotating drum for the study of aerosols over an extended period of time. Amer. J. Hyg. 68:85-93.

4. Harper, G. J., and J. D. Morton. 1952. Bacillus subtilis spores labelled with radio-phosphorus. J. Gen. Microbiol. 7:98-106.

5. Jemski, J. V., and G. B. Phillips. 1965. Aerosol challenge of animals, p. 285-295. In W. I. Gay (ed.), Methods of animal experimentation. Academic Press Inc., New York.

6. Koller, L. R. 1952 . Ultraviolet radiation, p. 1-2. John Wiley and Sons, New York.

7. Perkins, W. A., and L. M. Vaughan. 1961. Public health implication of airborne infection: physical aspects. Bacteriol. Rev. 25:347-355.

8. Webb, S. J. 1965. Bound water in biological integrity, p. 90-133. Charles C Thomas, Publisher, Springfield, Illinois.

9. Wolfe, E. K., Jr. 1961. Quantitative characterization of aerosols. Bacteriol. Rev. 25:194-202. 